\title{
Key indicators
}

The data in this table support the Economic review by providing some of the latest estimates of Key indicators.

Seasonally adjusted unless otherwise stated

$\begin{array}{lrrrrrrrr}\text { Source } & 2005 & 2006 & 2007 & 2007 & 2007 & 2007 & 2007 & 2007 \\ \text { CDID } & & & \text { Q1 } & \text { Q2 } & \text { Q3 } & \text { Aug } & \text { Sep } & \text { Oct }\end{array}$

GDP growth - chained volume measure (CVM)

ABMI

1.8

2.8

0.8

0.8

0.7

Output growth - chained volume measures (CVM)

\begin{tabular}{llrrrrrrr}
\hline Gross value added (GVA) at basic prices & ABMM & 1.9 & 2.8 & 0.8 & 0.8 & 0.7 &.. &.. \\
Industrial production & CKYW & -2.0 & 0.0 & -0.2 & 0.7 & 0.0 & 0.1 & -0.5 \\
Manufacturing & CKYY & -1.2 & 1.4 & -0.5 & 0.8 & 0.0 & 0.5 & -0.6 \\
Construction & GDQB & 1.5 & 1.1 & 0.8 & 0.7 & 0.8 &.. &.. \\
Services & GDQS & 2.9 & 3.6 & 0.9 & 0.9 & 0.9 &.. &.. \\
Oil and gas extraction & CKZO & -10.5 & -9.1 & 1.1 & 1.1 & -2.0 & -4.2 & 1.0 \\
Electricity, gas and water supply & CKYZ & -0.4 & -2.5 & 1.2 & -0.4 & 0.8 & -0.1 & 1.0 \\
Business services and finance & GDQN & 4.4 & 5.3 & 1.0 & 1.7 & 1.5 &.. &..
\end{tabular}

Household demand

\begin{tabular}{|c|c|c|c|c|c|c|c|c|c|}
\hline Retail sales volume growth & EAPS & 2.0 & 3.3 & 0.4 & 1.4 & 1.6 & 0.7 & 0.3 & -0.1 \\
\hline Household final consumption expenditure growth (CVM) & $A B J R$ & 1.5 & 2.0 & 0.7 & 0.8 & 1.0 & .. & .. & .. \\
\hline GB new registrations of cars (thousands) ${ }^{1}$ & BCGT & 2,444 & 2,340 & 678 & 573 & 671 & 76 & 419 & .. \\
\hline
\end{tabular}

Labour market ${ }^{2,3}$

Employment: 16 and over (thousands)

Employment rate: working age (\%)

Workforce jobs (thousands)

Total actual weekly hours of work: all workers (millions)

Unemployment: 16 and over (thousands)

Unemployment rate: 16 and over (\%)

Claimant count (thousands)

Economically active: 16 and over (thousands)

Economic activity rate: working age $(\%)$

Economically inactive: working age (thousands)

Economic inactivity rate: working age (\%)

Vacancies (thousands)

Redundancies (thousands)

$\begin{array}{lrr}\text { MGRZ } & 28,707 & 28,947 \\ \text { MGSU } & 74.7 & 74.6 \\ \text { DYDC } & 31,042 & 31,409 \\ \text { YBUS } & 918.6 & 923.7 \\ \text { MGSC } & 1,429 & 1,660 \\ \text { MGSX } & 4.7 & 5.4 \\ \text { BCJD } & 861.7 & 944.7 \\ \text { MGSF } & 30,135 & 30,607 \\ \text { MGSO } & 78.5 & 78.9 \\ \text { YBSN } & 7,939 & 7,851 \\ \text { YBTL } & 21.5 & 21.1 \\ \text { AP2Y } & 616.8 & 594.7 \\ \text { BEAO } & 126 & 145\end{array}$

29,053
74.3
31,602
929.5
1,705
5.5
916.3
30,759
78.8
7,954
21.2
636.8
145

29,154
74.4
31,689
937.5
1,660
5.4
877.1
30,814
78.8
7,964
21.2
647.5
120

$\begin{array}{rr}29,223 & 29,223 \\ 74.4 & 74.4 \\ . . & . . \\ 937.8 & 937.8 \\ 1,667 & 1,667 \\ 5.4 & 5.4 \\ 846.8 & 848.6 \\ 30,889 & 30,889 \\ 78.8 & 78.8 \\ 7,972 & 7,972 \\ 21.2 & 21.2 \\ 667.0 & 664.7 \\ 134 & 134\end{array}$

824.8

Productivity and earnings annual growth

GB average earnings (including bonuses) ${ }^{3}$

GB average earnings (excluding bonuses) ${ }^{3}$

Whole economy productivity (output per worker)

Manufacturing productivity (output per job)

Unit wage costs: whole economy

Unit wage costs: manufacturing

\section{LNNC}

JQDY

A4YN

LOUV

LOJE

LOJF

Business demand

Business investment growth (CVM)

NPEL

15.7

$-4.3$

$-0.5$

0.4

0.0

Government demand

Government final consumption expenditure growth

NMRY

2.7

2.1

0.5

0.3

0.3

Prices (12-monthly percentage change - except oil prices)

\begin{tabular}{|c|c|c|c|c|c|c|c|c|c|}
\hline Consumer prices index ${ }^{1}$ & D7G7 & 2.1 & 2.3 & 2.9 & 2.6 & 1.8 & 1.8 & 1.8 & 2.1 \\
\hline Retail prices index ${ }^{1}$ & $\mathrm{CZBH}$ & 2.8 & 3.2 & 4.5 & 4.4 & 3.9 & 4.1 & 3.9 & 4.2 \\
\hline Retail prices index (excluding mortgage interest payments) & CDKQ & 2.3 & 2.9 & 3.7 & 3.4 & 2.7 & 2.7 & 2.8 & 3.1 \\
\hline Producer output prices (excluding FBTP) ${ }^{4}$ & EUAA & 2.1 & 2.3 & 2.6 & 2.2 & 2.3 & 2.4 & 2.2 & 2.3 \\
\hline Producer input prices & EUAB & 11.6 & 9.7 & -0.8 & 0.9 & 2.6 & 0.7 & 6.7 & 8.5 \\
\hline Oil price: sterling (f per barrel) & ETXR & 30.36 & 35.93 & 29.95 & 34.05 & 36.93 & 35.47 & 38.09 & 40.38 \\
\hline Oil price: dollars ( $\$$ per barrel) & ETXQ & 55.05 & 66.11 & 58.53 & 67.64 & 74.67 & 71.36 & 76.92 & 82.60 \\
\hline
\end{tabular}

Oil price: dollars (\$ per barrel)

66.11

74.67

$\begin{array}{rrr}4.1 & 3.7 & 4.1 \\ 3.7 & 3.7 & 3.7 \\ . . & . . & . . \\ . . & 3.4 & 3.0 \\ . . & . . & . . \\ . . & 0.2 & 0.1\end{array}$


Seasonally adjusted unless otherwise stated

\begin{tabular}{lrrrrrrrr} 
Source & 2005 & 2006 & 2007 & 2007 & 2007 & 2007 & 2007 & 2007 \\
CDID & & & Q1 & Q2 & Q3 & Aug & Sep & Oct \\
\hline
\end{tabular}

\section{Financial markets}

Sterling ERI (January 2005=100)

Average exchange rate /US\$

Average exchange rate /Euro

3-month inter-bank rate

Selected retail banks: base rate

3-month interest rate on US Treasury bills

BK67

AUSS $\quad 1.8197$

THAP

HSAJ

ZCMG

LUST

1.4629

4.57

3.92

101.2
1.8429

1.4670

5.26

104.6

1.9546

1.4916

5.56

4.89

4.91

$\begin{array}{rr}104.1 & 104.1 \\ 1.9870 & 2.0211\end{array}$

$1.4732 \quad 1.4705$

5.93

6.18

4.68

3.62

104.3
2.0111
1.4762
6.55
5.75
3.91

103.0

2.0185

1.4515

6.18

5.75

Trade and the balance of payments

\section{UK balance on trade in goods ( $\mathrm{fm}$ )}

Exports of services ( $\mathrm{fm}$ )

Non-EU balance on trade in goods ( $\mathrm{fm}$ )

Non-EU exports of goods (excl oil \& erratics) ${ }^{5}$

Non-EU imports of goods (excl oil \& erratics)

Non-EU import and price index (excl oil) ${ }^{5}$

Non-EU export and price index (excl oil $)^{5}$

BOKI $\quad-68,789 \quad-77,563$

IKBB 115,182

LGDT $\quad-31,912$

124,586

$-20,605$

$-20,346$

\section{$-20,346$}

$-22,257$

33,993

$-11,451$

$-10,724$

$-13,108$

$-45,587$

SHDJ $\quad 119.8$

SHED $\quad 116.8$

118.0

115.2

115.3

117.3

134.5

$\begin{array}{lll}\text { LKWQ } & 101.2 & 103.9\end{array}$

$126.5 \quad 128.3$

$104.6 \quad 104.5$

$101.9 \quad 101.9$

103.4

102.2

$-6,948$

10,976

$-3,953$

121.9

131.4

103.5

101.8

\section{$-7,754$}

10,922

$-4,711$

114.2

133.6
103.7

103.7

\section{Monetary conditions/government finances}

Narrow money: notes and coin (year on year percentage growth) ${ }^{6}$

M4 (year on year percentage growth)

Public sector net borrowing ( $\mathrm{fm}$ )

Net lending to consumers ( $\mathrm{fm}$ )

$\begin{array}{lr}\text { VQUU } & 3.1 \\ \text { VQJW } & 11.3 \\ \text {-ANNX } & 40,7 \\ \text { RLMH } & 19,7\end{array}$

$3.1-5.0$

4.1
12.6
-2.6

4.8
12.9
15.873

$\begin{array}{rrrr}5.4 & 4.6 & 5.4 & . . \\ 12.8 & 13.5 & 12.8 & . . \\ 9,276 & 8,417 & 7,151 & -993 \\ 3,677 & 1,114 & 1,350 & . .\end{array}$

External indicators - non-ONS statistics

\begin{tabular}{rrrrrrrrr}
\hline & 2007 & 2007 & 2007 & 2007 & 2007 & 2007 & 2007 & 2007 \\
Nov & Apr & May & Jun & Jul & Aug & Sep & Oct & Nop
\end{tabular}

\section{Activity and expectations}

\begin{tabular}{|c|c|c|c|c|c|c|c|c|c|}
\hline CBI output expectations balance & ETCU & 18 & 18 & 25 & 10 & 13 & 17 & 10 & 9 \\
\hline CBI optimism balance & ETBV & 16 & & & -2 & & & -13 & \\
\hline $\mathrm{CBI}$ price expectations balance & ETDQ & 14 & 26 & 18 & 17 & 17 & 20 & 15 & 22 \\
\hline
\end{tabular}

\section{Notes:}

1 Not seasonally adjusted.

2 Annual data are for April except for workforce jobs (June), claimant count (average of the twelve months) and vacancies (average of the four quarters).

3 Monthly data for vacancies and average earnings are averages of the three months ending in the month shown. Monthly data for all other series except

claimant count are averages of the three months centred on the month shown.

4 FBTP: food, beverages, tobacco and petroleum.

5 Volumes, $2003=100$

6 Replacement for series M0 which has ceased publication.

For further explanatory notes, see Notes to tables on page 63. 